(c) 2010 IEEE. Personal use of this material is permitted. Permission from IEEE must be obtained for all other uses, in any current or future media, including reprinting/republishing this material for advertising or promotional purposes, creating new collective works, for resale or redistribution to servers or lists, or reuse of any copyrighted component of this work in other works. 


\section{Adaptive Channel Coding and Modulation Scheme Selection for Achieving High Throughput in Wireless Networks}

\author{
${ }^{1}$ Atif Sharif,${ }^{2}$ Vidyasagar M. Potdar \\ Digital Ecosystems and Business Intelligence Institute \\ Curtin University of Technology \\ Perth, Western Australia \\ 12atif.sharif@postgrad.curtin.edu.au, \\ ${ }^{2}$ v.potdar@curtin.edu.au
}

\author{
${ }^{3}$ Rana Fayyaz Ahmad \\ Digital Ecosystems and Business Intelligence Institute \\ Curtin University of Technology \\ Perth, Western Australia \\ ${ }^{3}$ fayyaz@aero.com.pk
}

\begin{abstract}
Modern wireless communication demands reliable data communication at high throughput in severe channel conditions like narrowband interference, frequency selective fading due to multipath and attenuation of high frequencies. Traditional single carrier systems address this set of problems by the use of complex, computationally intensive equalization filters. The Orthogonal Frequency Division Multiplexing (OFDM) based system, as opposed to single-carrier systems, is considered to be the future of the wireless communication and is being used to achieve high data rate by overcoming severe channel conditions without the use of these complex filters. This paper discusses the problem of Adaptive Modulation scheme selection through an OFDM based system over parallel frequency selective fading channels. An adaptive coding scheme is proposed by using Generalized Concatenated Codes (GCC), which have simple structure and are designed in such a way that they are best suited for fading channels. GCC are based on binary cyclic codes. The criterion of the proposed research is to optimize the throughput of a wireless system. Depending on the quality of sub-channels an adaptive modulation selection scheme and code assigning method is proposed. The proposed research combats against channel impairments better than those used in conventional systems by exploiting individual sub-channel condition. Results show better performance in terms of higher throughput by minimizing the bit error rate.
\end{abstract}

Keywords- GCC, Channel State Information (CSI), OFDM, COFDM, Fading.

\section{INTRODUCTION}

Wireless communication technology, a fundamental part of modern information infrastructure, is growing day by day. Spectrally efficient and reliable transmission schemes are used for high speed transmission data rate over wireless link. However reliable data communication is one of the major problems in modern wireless channels, since these channels should be able to tolerate the effects of signal fading channels and Inter Symbol Interference (ISI). Mobile data communication is even more challenging. It requires continuous measurement and updating the Channel State Information (CSI) and adaptation of the coding/modulation techniques according to the new environmental conditions. It also involves proper power distribution techniques [1].
Channel coding is one of the main tools that increase the transmission reliability at higher data rates. The purpose of the channel coding scheme is to guard the information against channel disturbances, achieved by introducing data redundancy for error detection and correction. In wireless communication systems erroneous signal detection and its correction can be achieved by the use of proper error control coding techniques. The bursty traffic sources which are multiplexed statistically, the fast and dynamic channel capacity allocation can be achieved by Single-carrier Quadrature Phase Shift keying (QPSK) or Quadrature Amplitude Modulation (QAM) [18]. However such single-carrier systems show their effectiveness in severe multipath applications requiring intensive processing at high data rates [18] only if they use adaptive equalizer. Another problem with single carrier systems operating is susceptibility to interference.

\section{A. Introduction to OFDM}

Orthogonal frequency division multiplexing (OFDM) sometimes called multi-carrier or discrete multi-tone modulation used for digital TV in Japan, Europe and Australia. OFDM, being spread spectrum by nature, uses a number of carriers, which are spectrally distributed at precise frequencies, for data transmission. Orthogonality is being provided by this spectrum spacing which prevents demodulator interference with other frequencies. OFDM offers a number of benefits like resiliency to Radio Frequency (RF) interference, high spectral efficiency, and lower multi-path distortion. The increasingly need for high speed mobile data transmission is a byproduct of the recent advancements in the digital wireless communication. To counter this increasingly need for high speed data transmission capacity researchers are continually engaged in envisaging new modulation techniques. As power is the biggest concern in most of the wireless communication, OFDM has feasibly gained its high processing power at economical cost in comparison to existing wireless technologies. And now OFDM is considered as major technological contender for $4 \mathrm{G}$ wireless applications. DSL, HDTV broadcasting, DAB, IEEE802.11 are some of the key examples of applications involving OFDM for high rate wireless communication. In OFDM high bandwidth efficiency is achieved by using the orthogonal tomes for signal modulation. The tones are evenly spaced at defined intervals, 
equal to symbol rate, in frequency band and can be easily separated at the receiving end. In case of mobile wireless channels multi path delay is a serious issue and is being handled effectively by OFDM which simultaneously handles the symbol duration and data transmission in parallel. The channel capacity in case of OFDM based system is increased due to the fact that OFDM splits the data bearing the radio signal into multiple data blocks. Each data block in turn being modulated by the different sub carriers which are orthogonally spaced in frequency spectrum thereby preventing the interference occurred during the simultaneous transmission over wireless channel. In OFDM the spacing between the sub carriers is chosen in such a way that they are all orthogonal to each other. In [14] researchers envisaged a method for maintaining this orthogonality. According to the method the sub carrier's frequency spacing is chosen to the inverse of the sub carrier symbol duration.

The rest of the paper is organized as follows. Section II lists some of the related work followed by section III where we have outlined the problem definition. The Design parameters and the conceptual framework are discussed in the Section IV and V respectively. Section VI shows the effectiveness of the proposed scheme followed by the Section VII where we have discussed the future research directions and finally the conclusion of the paper is drawn in Section VIII.

\section{RELATED WORK}

Concatenated codes are used to obtain long and powerful codes by using simple constituent codes. Product codes are a kind of concatenated codes and are presented by Elias [3]. They can be represented as a set of matrices such that each row in these matrices is a codeword in one constituent code and each column is a codeword in another constituent code. One important property of product codes is burst error correction.

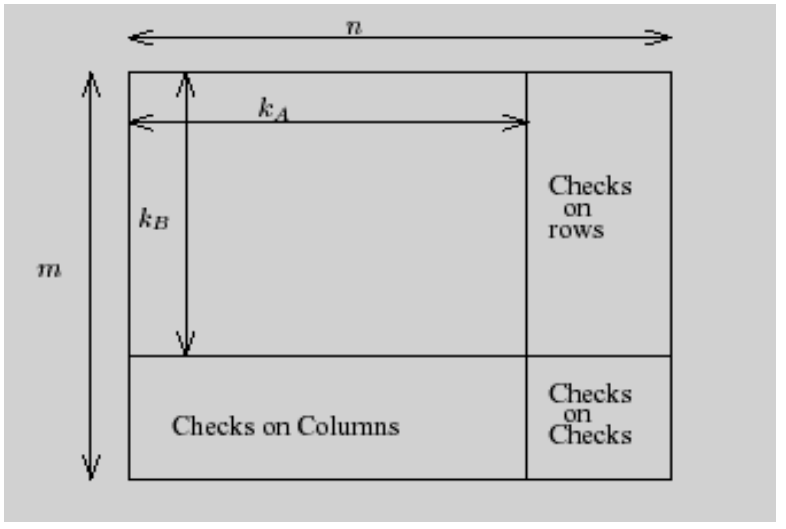

Figure 1. Structure of Product Codes [1]

Product codes are serially concatenated codes [12]. The idea of product codes is quite simple and powerful at the same time where very long block codes can be constructed by the use of two or more shorter constituent codes. Consider A' and B' as two block codes with performance parameters $\left[n, k_{A}, d_{A}\right]$ an $\left[\mathrm{m}, \mathrm{k}_{\mathrm{B}}, \mathrm{d}_{\mathrm{B}}\right.$ ], respectively [1]. The rates of the codes $\mathrm{A}^{\prime}$ and $\mathrm{B}^{\prime}$ are denoted by $\mathrm{R}_{\mathrm{A}}$ and $\mathrm{R}_{\mathrm{B}}$, respectively, and are equal to:

$$
\begin{gathered}
R_{A}=k_{A} / n \\
R_{B}=k_{B} / m
\end{gathered}
$$

The product code $\mathrm{C}$ is obtained from the codes $\mathrm{A}^{\prime}$ and $\mathrm{B}^{\prime}$ in the following manner:

1. Place $\mathrm{kA} \times \mathrm{kB}$ information bits in a metrics having $\mathrm{kA}$ columns and $\mathrm{kB}$ rows.

2. Code $\mathrm{A}^{\prime}$ will be used to code $\mathrm{kB}$ rows resulting in an array having $\mathrm{n}$ columns and $\mathrm{kB}$ rows.

3. Code B' will then be used for coding the $\mathrm{n}$ columns.

The construction of the product code $\mathrm{C}$ with constituent codes $\mathrm{A}^{\prime}$ and $\mathrm{B}^{\prime}$ is illustrated in Figure 1 [1].

The parameters of the resulting product code will be [mn, $\mathrm{kAkB}, \mathrm{dAdB}$ ] and its rate will be equal to RARB. Therefore, we can construct long block codes starting by combining two short codes. Product codes have much smaller minimum distance as compared to optimal codes. The error correcting potential of product codes is quite large. They have burst error correction capability. The covering radius of product codes is, usually, much greater than half the minimum distance of the code [10]. Forney was the first to propose concatenated codes [4]. The aim was to find a code that approaches the channel capacity with practical decoding complexity that increase polynomially with the length of the code [11]. The proposed code was a concatenation of a relatively short inner code with an outer long Reed-Solomon code [12].A very important characteristic of concatenated codes is the Hamming weight distribution [19] of the codes and their Euclidean distance spectrum [17]. These characteristics are very important for determining the error correction capability of the codes by using different bounds. Generalized concatenated codes are further generalization of concatenated codes. They can be viewed as binary matrices with the rows and columns belonging to many different block codes. They were introduced by Zinoviev [5]. Concatenated codes are efficient in wireless communication channels because they have comparatively high minimum distances and they have proper structure for burst error correction without the need for extra interleaving. Interleaving is used to transform burst errors into random errors which then can be corrected by forward error control codes. Concatenated codes have higher reliability with slight increase in complexity. By using concatenated codes different data rates can be achieved as several different codes are used for encoding the rows and columns instead of restricting oneself to only one code for the rows and one code for the columns. Adaptive modulation has also recently gained momentum in wireless systems because it provides more efficient use of channel. For OFDM systems the researchers in [7] and [13] investigated the adaptive modulation criteria. The proposed research in [9] had taken the time varying channel conditions for adaptive modulation and coding. For OFDM systems, the adaptive modulation is investigated according to Average Channel Conditions by [8]. The idea of application in OFDM system is presented in [2]. For OFDM systems, researcher in [1] had proposed an adaptive coding scheme that uses generalized concatenated codes. The proposed coding scheme was applied to HIPERLAN/2, whose performance was then analyzed against the standard convolution coding scheme in 
use. The objective of research was to enhance the system's throughput and was achieved by adaptively selecting the modulation and channel coding schemes based on varying subchannels conditions.

\section{PROBLEM DEFINITION}

The modern wireless communication demands reliable data communication at high throughput in severe channel conditions like narrowband interference, frequency-selective fading due to multipath and attenuation of high frequencies. The existing Single carrier systems address the above mentioned problems by involving complex and computationally intensive channel equalizers. Also reliable data transmission and security is of major concern these days. Thus a need to make more efficient use of bandwidth transmissions without creating situations where signals would be subject to above mentioned phenomenon is generally desired. In order to achieve these design objectives of wireless data communication there are some key design considerations like channel coding, Multiple access schemes, adaptive modulation, rate adaptive transmission etc which must be taken into considerations and are discussed in the following section.

\section{DESIGN CONSIDERATIONS}

Following parameters must be considered while designing the wireless data communication system, these are

\section{Channel Coding}

2. Multiple access Schemes

\section{Adaptive Modulation}

\section{Rate-adaptive Transmission}

\section{Severity of Wireless Communication}

Channel coding is also considered as an essential and important element of communication system, one of the main tools that increase the transmission reliability at higher data rates. The purpose of the channel coding scheme is to guard the information against channel disturbances during transmission, and this can be achieved by introducing data redundancy for error detection and correction. It protects data against transmission errors to ensure adequate transmission quality (bit or frame error rate). It is power efficient as compared to the uncoded case, the same error-rates are achieved with much less transmit power at the expense of a bandwidth expansion. Error control coding has gained fundamental importance in wireless communication systems and deals with techniques for detecting and correcting signal errors.

Multiple access schemes narrow or wide band, are a very important means of allowing a large number of mobile users to access a finite amount of frequency spectrum simultaneously. By doing this capacity can be increased. In simple words they ensure the efficient use of the frequency spectrum. They are also preferred over Radio spectrums since they are very expensive.

Adaptive modulation schemes take channel conditions into account at both the transmitter and receiver end. And this knowledge of channel conditions helps transmitter and receiver in mutually deciding the channel modulation scheme for gaining increased performance which results in high data rate as compared to non adaptive coded schemes.

Rate Adaptive Transmission design feature relies more on the quality of the wireless channel and it ensures the maximal use of the transmission bandwidth. It achieves high spectral efficiency by increasing the sending information rate during the good channel conditions and vice versa. Channel status in form of channel state information (CSI) is being sent by the receiver to the transmitter on the reverse wireless link. This information will then be used by the transmitter in deciding the rate plan for the next transmission. This rate adaptive transmission ensures the maximal utilization of the channel bandwidth for an acceptable Bit-Error-Rate (BER).

The Severity of Wireless Communications is much greater than that for AWGN channels. For AWGN channels, good codes can be designed by making the distance between the code words as large as possible, or, alternatively, to make the number of code words at small Euclidean distance as small as possible. But, for fading channels, the severity of channels is mainly tackled by the diversity in communication. Diversity in communication means sending a copy of the message, or part of a copy, on different paths or channels in order to increase the reliability. It can be achieved by diversity in carrier frequency, space diversity, by coding or a combination of all methods.

\section{PROPOSED GENERALIZED CONCATENATED CODE SCHEME}

In this paper, the problem of Adaptive Coded Modulation through an OFDM based system over parallel frequencyselective fading channels using Generalized Concatenated Codes (GCC) is proposed. These codes are simple by structure and rely on the concatenation of two or more shorter length codes. The large diversity design feature of such codes makes them choice of interest for use in case of channels suffers from fading also called fading channels. GCC are based on binary cyclic codes. The decoding of the proposed codes is performed by GMD decoding. These codes can be used in current wireless systems where approaching channel capacity is, usually, of less importance than the processing delay, energy consumption or integrated circuit chip size [1]. The method used for designing the codes bear great similarities to the ideas presented by in [1] with some modifications that take into consideration the channel conditions.

\section{A. Proposed Coded OFDM (COFDM)}

In this Paper, COFDM is used. First the channel is subdivided into $\mathrm{N}$ number of sub-channels. Depending upon the channel state information of the sub-channels the carrier to interference ratio and the AWGN noise is calculated. Based on this information the modulation and the encoding are preformed. Thus the COFDM exploits the channel state information in order to achieve the best results that would ensure the highest throughput. Here, the concept of multiple accesses in OFDM is exploited to accommodate multiple codes with data rates that vary in coding and in modulation. Thus it would be adaptive multiple access on the OFDM. The following points taken in consideration 
1. A coding scheme, generalized concatenated codes, with good error correction capabilities especially in fading channels.

2. Choosing optimum Coding dimensions according to the individual channel conditions and Proper selection of codes for row blocks.

3. Designing an effective adaptive coded modulation algorithm for each sub-channel such that throughput is maximized, while keeping the complexity of the system to a minimum.

4. Generalized Minimum distance (GMD) decoder will be used for decoding. In the proposed scheme the criterion of subchannels quality is being used in defining the adaptive modulation scheme selection and the corresponding information coding scheme that adapts according to the varying wireless channel conditions. The proposed research offers significant advantage in protecting the information as compared to conventional schemes that did not take into account the conditions of individual wireless channel. The criterion of study is the optimization of the throughput of the system, while maintaining the PER (Packet Error Rate).

\section{B. System Overview}

Instead of the average standardized one, the structure of the proposed GCC code has taken into account the dependency of individual sub-channels' condition. It is shown in Figure.2 [1].

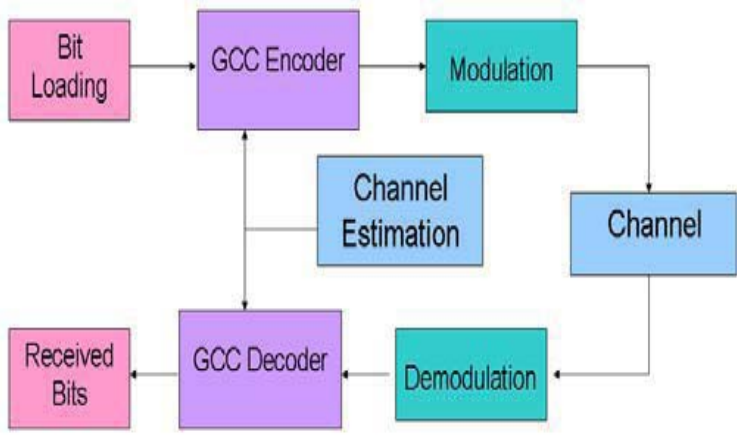

Figure 2. System Structure [1]

\section{Channel Estimation:}

In order to select the best possible parameters we need to know the Channel State Information. This CSI will determine the criteria that would allow us to select the right parameters for transmitting the data. This CSI has to be reliable in order to produce good results. In wireless communication systems, the channel estimation is performed by transmitting a training sequence [20] [21], i.e., a sequence of symbols that are known to the receiver in advance. This sequence of symbols is used to estimate the parameters of the channel. There are basically two aspects which are taken into account while system structuring these includes

A) Choosing different modulation scheme for each sub channel

B) For each individual sub-channels a decision on selecting the codes blocks
The above two aspects are interrelated in the sense that the choice of one effects the others and vice versa. The criterion is to maximize system's throughput by maintaining probability of error.

\section{1) Modulation Scheme}

The choice of modulation scheme plays a very important role. This is so because it would be a balancing act between the throughput and the probability of error. This means that the order of modulation needs to be selected in such a manner that the maximum allowable probability of error is never exceeded. However, the catch is the throughput must be maximized. When this is done, there would be a compromise with the probability of error. Depending upon the (Channel State Information) CSI the best possible modulation scheme is selected. The following discussion will focus upon the fact that the CSI has been calculated; and the proper modulation order and type need to be predicted. The modulation schemes that are used here are discussed in this section. Each sub-channel is Rayleigh in nature. So the processing of sending data on any one of the channel can be represented by the multiplication of the data and the channel state information and then adding the AWGN noise. This process can be represented by the following equation

$$
r(t)=c(t) \cdot s(t) \cdot n(t)
$$

Where, $\mathrm{r}(\mathrm{t})$ is the received signal, $\mathrm{c}(\mathrm{t})$ is the Channel State Information, $\mathrm{s}(\mathrm{t})$ is the data signal and is the AWGN noise. This Channel State Information (CSI) and the AWGN noise is used to determine the modulation order. For this, the waterfilling algorithm is used to calculate the Carrier-to-Interference ratio. This Carrier-to-Interference ration will used to calculate the theoretical probability of error. There are four types of modulations used here. These methods are
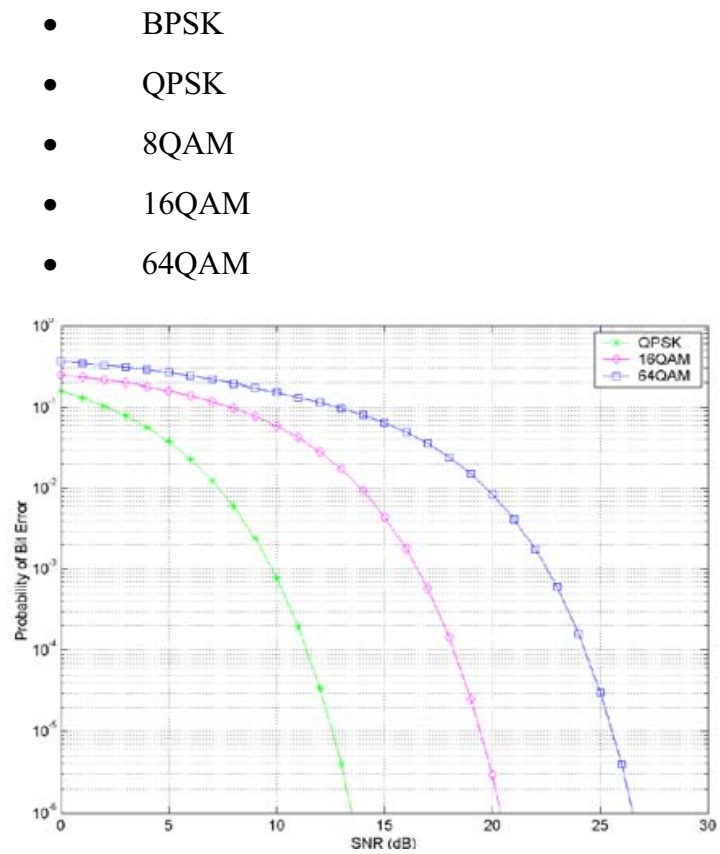

Figure 3. Bit Error Probability of Channel 
Using the above equation (2.2) their behavior can be depicted in the Figure 3. From this graph we need to choose a point that will serve as threshold point for the maximum allowable Bit Error Rate. From the above figure, we can see that QPSK is most robust of them all. Therefore, by choosing a maximum probability of error for this method will provide us with a threshold for a minimum operate able SNR. In our case let the maximal allowable rate is $10^{-3}$.

This performance would be possible only for the SNR greater then $10 \mathrm{~dB}$ at least. From the graph it can be seen that there is no modulation method that would be able to perform better than the QPSK for a given probability of error. Therefore we will assign this method whenever the channel is very bad and there is no possible way for the increase of throughput without compromising the system performance with respect error probability. The choice of these modulation methods depends on two reasons

1. Their ability to provide higher throughput with acceptable bit error rate

\section{Their contribution to the construction of GCC's}

Each sub-channel will be assigned one of these modulation schemes. This process depends a lot on each sub channel's Channel State Information (CSI). The Water-Filling algorithm to compute the Carrier-to-Interference ratio $(\mathrm{C} / \mathrm{I})$ will use the CSI. Thus $(\mathrm{C} / \mathrm{I})$ of each sub-channel will provide the criteria for the selection for the method that would provide us with the best throughput. The carrier to interference ratio will provide us an upper limit on the probability of error for each method. For this the following formulae are used:

$$
\begin{aligned}
& P b_{t h}=Q\left(\sqrt{2 * \frac{C}{I}}\right) \text { forBPSK } \\
& P b_{t h}=Q\left(\sqrt{\frac{C}{I}}\right) \text { for } Q P S K \\
& P b_{t h}=\left(\frac{3}{4}\right)\left(Q\left(\sqrt{2 * \frac{C}{I}}\right)\right) \text { for } 16 Q A M \\
& P b_{t h}=\left(\frac{7}{12}\right)\left(Q\left(\sqrt{2 * \frac{C}{I}}\right)\right) \text { for } 64 Q A M
\end{aligned}
$$

These formulae will give us the theoretical bound on the probability of error. This bound is computed for each subchannel for each of these methods. Once this is computed then a comparison is preformed in order to determine which modulation scheme to be used. For this purpose the practical probability of error is calculate for every sub-channel using each of the above mentioned modulation schemes. After this is done, a comparison is preformed. In this comparison, the method that would yield the maximum throughput is selected. In the worst case scenario QPSK would be selected so as the probability of error never exceeds the allowable level. So in order to select the right modulation scheme the following criteria

$$
P b \leq P b_{t h}
$$

where the practical probability of error is $P b$.

\section{2) Coding Scheme}

Channel coding is used in communication systems to eliminate or greatly reduce the errors that are introduced by the channel, so that a reliable communication can be possible. All codes are, basically, a pre-selected subset of sequences from the total space of signal sequences. A simple GCC type coding scheme is implemented where the row vector will be loaded by the different information bits according to the code used. After this different binary Bose-Chaudhuri-Hochquenghem $(\mathrm{BCH})$ codes will be applied to achieve the row encoding. Each row code is subset of each other. After the row encoding the one binary $\mathrm{BCH}$ code will be used to perform the column encoding. Figure 3 depicts the structure of the GCC used in this system, where the colored and shaded area points to the information and redundancy bits. The information required in order to build the Generalized Concatenated Code is the modulation scheme. Since in our system OFDM is used there will be N number of sub-channels. Each of these sub-channels will have its own modulation scheme. The algorithm for the adaptive selection of the modulation schemes adaptively computes this modulation scheme. Once it has done, we have the following information

- $\quad$ Method of Modulation

- Order of Modulation

- $\quad$ Numbers of sub channels

\section{3) Algorithm}

The following algorithm will demonstrate how the modulation scheme is selected for each of the sub-channels along with the respective rates. The process of power allocation is also covered within these steps. The proposed concept is summarized below.

a) STEP 1: POWER DISTRIBUTION AMONG SUBCHANNELS.

1. Compute the SNR of each sub-channel as Carrier-tointerference ratio $\mathrm{C} / \mathrm{I}$

2. Compute the initial power distribution for all the subchannels

3. Check if any of the sub-channels is allotted negative power. If not then follow to the next step otherwise follow the following procedure:

a) Those indexes that have a positive value of power will be marked.

b) These marked indexes will refer to those values of $\mathrm{C} / \mathrm{I}$ that correspond to positive values of sub-channel power. Thus power will be redistributed among these marked C/I subchannels while ignoring those with negative power.

c) This negative power test is repeatedly preformed until no negative power is assigned to any channel.

d) If there is no more negative sub-channel power then go to the step (following) 4 . 
e) The computed values of $\mathrm{C} / \mathrm{I}$ and initial values of allocated power per sub-channel are set as final values for further computation.

b) STEP 2: COMPUTE Pb FOR EACH SUB-CHANNEL:

Using the computed $\mathrm{C} / \mathrm{I}$ of each sub-channel the empirical values of the probability of error is computed. Along with this the practical probability of error is also computed for each subchannel. The above steps are preformed for each of the four modulation schemes.

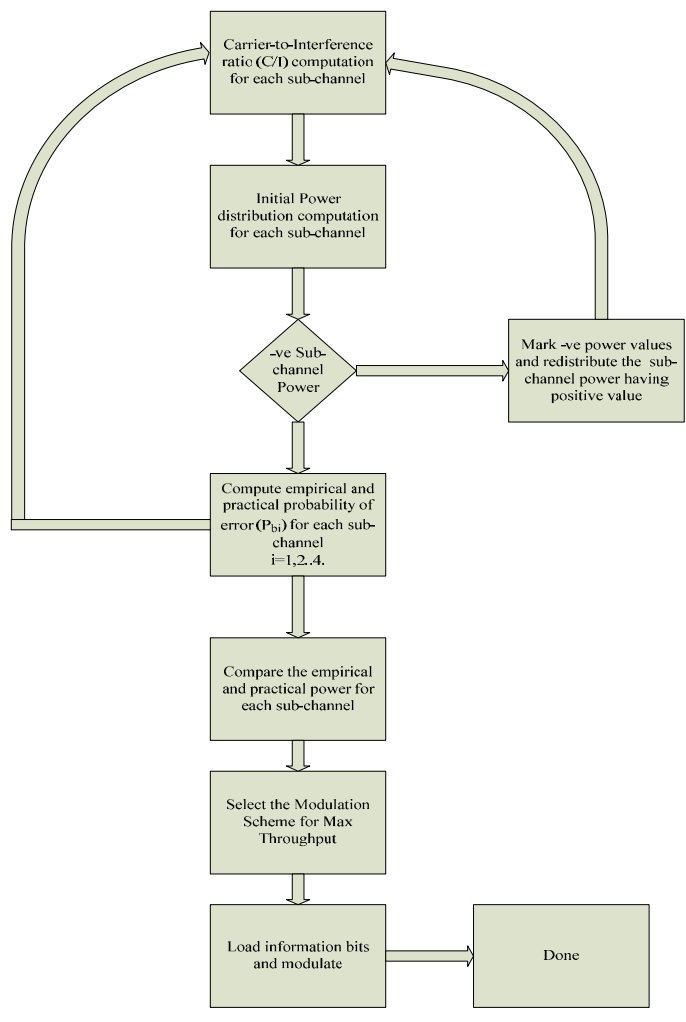

Figure 4. Algorithm

c) STEP 3: CODING AND MODULATION SCHEME ASSIGNMENT PER SUB-CHANNEL:

Compare the empirical and practical values of error probability for each sub-channel selecting the best scheme for maximization of throughput Based on the modulation scheme select respective coding scheme.

d) STEP 4: LOAD THE INFORMATION BITS AND MODULATE.

\section{RESULTS}

The main idea while erecting the simulation, that uses Matlab as simulation environment, to support the acclamations, the main idea was to keep the whole process as simple as possible. At the same time, it was also essential to imitate the reality as much as possible. This purpose involved some assumptions. It was understood that there was a perfect knowledge of sub-channels conditions.

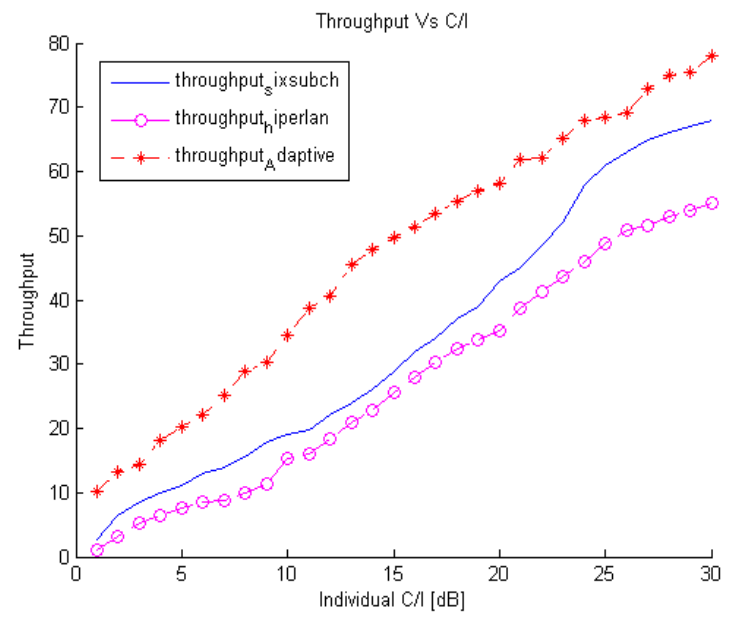

Figure 5. Comparison of Throughput

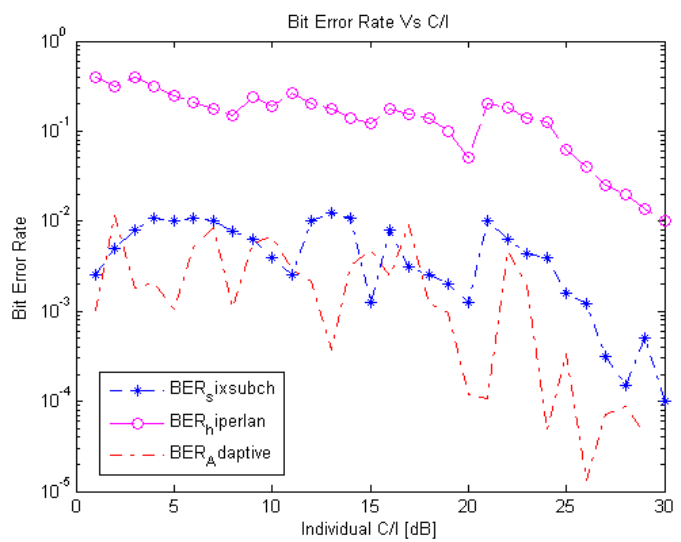

Figure 6. Comparison of BER

In this paper after a large number of iteration the set of horizontal encoders that was selected are $k_{A}=$ $\{30,36,39,45,51,57\}$ and for the vertical encoder $k_{B}=57$ and $n$ $=\mathrm{m}=63$. Here, we seeked no optimum solution by the use of sub-channels for redundancy. Figure 5 depicts the throughput comparison of the proposed idea and standardized $\mathrm{H} / 2$ one [15]. It also figures out the case of six sub-channels for redundancy [1]. The BER is depicted in Figure. 6, here discontinuities are a result of the change of the modulation scheme used.

It is worth mentioning that high bandwidth, due to frequent reporting processes, for reverse link will be required in the proposed improvement scheme. Additionally the process complexity measured in terms of number of cyclic operations per decoding, is nearly comparable to the conventional decoding process used in commercial OFDM systems [16].

\section{A. Comparisons between Referenced and Proposed Predicament}

The Figures 5 and 6 illustrates that the referenced adaption of $\mathrm{H} / 2$ shows better performance in terms of throughput, while 
sustaining a low PER. It is also possible to attain a certain throughput at low power there by resulting in less interference and hence increased system's capacity. Also the proposed enhancement outperforms $\mathrm{H} / 2$ in certain applications which are subject to tight error constraints $(<1 \%)$. The decoding process complexity is measured in terms of the cycles/operations it would take to decode, is much less or at worst as compared to the conventional decoding process used in commercial OFDM systems. Here, the throughput is maximized while attaining the minimum probability of error. Therefore, as in general the proposition was able to obtain an optimally maximized throughput.

\section{DISCUSSION/ FUTURE WORK}

In this paper, we have used five types of modulations namely BPSK, QPSK, 8QAM, 16QAM and 64QAM. These five modulation schemes are used for the best utilization of channels. In reference paper [1] number of rows in GCC was equivalent to number of channels, so there was not enough redundancy added. In proposed work, numbers of row codes are equivalent to the number of channels, so enough redundancy is added to protect data from any corruption. So here data is better protected as compared to the conventional systems. In general we have used the Adaptive Modulation scheme selection in this paper in order to use each channel condition to its best, while maintaining the acceptable level of probability of error and maximizing the throughput. Thus, resources are not wasted and the COFDM is more efficiently used. This is because the adaptive modulation not only increases the throughput but also helps to encode the OFDM to better suit the channel conditions i.e. the Channel State Information, Carrier-to-Interference ratio and the SNR of each sub-channel. From the figures shown in result section, the proposed predicament that the results of the modification are better in both aspects that is the throughput and the probability of error as well. Thus the modification better utilizes the channel condition. We have been able to achieve these results because in state of deep fading the transmission would shutdown. Since a main focus was to improve our throughput; it was observed that whenever the channel was in good condition the modulation scheme with the highest rate would be selected while maintaining the acceptable probability of error. This in turn will adaptively select a coding scheme for each sub-channel. Here, the process complexity measured in terms of number of cyclic operations per decoding, is much less or at worst as compared to the conventional decoding process used in commercial OFDM systems [15]. Here number of row codes are equivalent to number of channels, therefore more redundancy is added. So the data is better protected against errors as compared to the conventional systems. Here the arrangement of the sub-channels in a particular order is not required. Also there is no need to have a particular code arrangement. Here adaptive coding and adaptive selection of modulation scheme according to individual channel conditions results in better performance of GCC. GCC is build up on linear binary codes and provide good performance under burst error conditions by eliminating the need of an interleaver/deinterleaver. These codes also proved to perform well in fading channels.

\section{CONCLUSION}

Proposed algorithm result in minimum system complexity and results in great performance gains as compared to conventional systems. Here, during the deep fading of the channel, transmission is closed. So it is possible to obtain some gain in total rate by switching off the transmission when the quality of the channel degrades below a certain level. This is a simple form of adaptation similar to that presented by Goldsmith et al in [6]. However, it is maintained that the net gain in rate is very small especially for high signal to noise ratios which is the same conclusion in [6].GMD decoding of generalized concatenated codes requires very low complexity. This decoding method decodes all errors of weight less than half the minimum distance. GMD decoding performs very well if the minimum distance of the concatenated code is very large, i.e., close to the maximum possible value. However, if the minimum distance is not very large, most of the error correction potential lies in correcting error patterns slightly greater than half the minimum distance [1]. Proposed algorithm result in minimum system complexity and results in great performance gains as compared to conventional systems.

\section{REFERENCES}

[1] Omar-Al-Askary et al, "Coding and iterative Decoding of Concatenated Multi-level Codes for Rayleigh Fading Channel," Doctoral Project in Radio Communication Systems, S3, KTH, Stockholm, Sweden, 2006

[2] L. Hanzo et al, "Adaptive Multicarrier Modulation: A Convenient Framework for Time-Frequency Processing in Wireless Communications," IEEE Proceedings of the IEEE 88, May, 2000

[3] P. Elias. "Error-free coding," IEEE Transactions on Information Theory, 4:29-37, 1954.

[4] David G. Forney Jr. "Concatenated Codes," The M.I.T press, Cambridge Massachusetts, 1966

[5] V. A. Zinov'ev. “Generalized cascade codes,” 1976.

[6] A. Goldsmith and P. Varaiya. "Capacity of fading channels with channel side information". IEEE Transactions on Information Theory, 43:19861992, Nov. 1997.

[7] O. A. Al-Askary et al, "Subcarrier based adaptive modulation in hiperlan/2 system," In 2004 IEEE International Conference on Communications, pages 3460-3464. IEEE, IEEE, Jun. 2004.

[8] Omar Al-Askary et al , "Adaptive Coding for OFDM Based Systems using Generalized Concatenated Codes," Radio Communication Systems, S3, KTH, Stockholm, Sweden

[9] H. Matsuoka et al, "Adaptive modulation system with variable coding rate concatenated code for high quality multimedia communication systems," Vehicular Technology Conference, Mobile Technology for the Human Race, IEEE 46th, pages 487-491. IEEE, IEEE, Apr. 1996.

[10] G. Cohen et al "Covering Codes," North Holland Math. Library, 1997.

[11] David G. Forney Jr. "Performance and complexity," In 1995 IEEE International Symposium on Information Theory, page 1, IEEE, IEEE, ep. 1995.

[12] Florence Jessie MacWilliams et al, "The Theory of Error-Correcting Codes," North-Holland, 1977.

[13] L. Zhen et al " Link adaptation of wideband OFDM systems in multipath fading channel," In proc. of IEEE Canadian Conference on Electrical and Computer Engineering, CCECE 2002, IEEE, May 2002, pp.1295- 1299 .

[14] Teng Li and M. Collins. "Capacity and Coding of Flat Fading Channels without Channel State Information”Department of Electrical Engineering University of Notre dame. Notre Dame, IN, 46556

[15] Johan Malmgren. "Product code implementation in an EDGE like system: Decoding and performance", M.sc., project, KTH, radio systems, May 2003. 
[16] P.Bansal and A.Brzezinski. "Adaptive Loading inMIMO/OFDM Systems" December 13, 2001

[17] MADDOCKS, M.C.D. "An introduction to digital modulation and OFDM techniques", BBC Research Department Report No. RD 1993/10, 1993.

[18] M. Sellars and D. Kostas, "Comparison of QPSK/QAM OFDM and Spread Spectrum for the 2-11 GHz PMP BWAS", In response to the Call For Contributions, by IEEE 802.16.3-00/07r1, 2000.

[19] J. D. Parsons. "The Mobile Radio Propagation Channel". JohnWiley and Sons, second edition, 2000.

[20] G. Forney. Us- patent \#1971000179653: “Training adaptive linear filters", Mar.1973.

[21] S. Qureshi. "Fast start-up equalization with periodic training sequences". IEEE Transactions on Information Theory, 23(5):553 - 563, Sep. 1977. 